\title{
Influence of Working Pressure on The Magnetic Properties of $\mathrm{Tb}\left(\mathrm{Fe}_{0.55} \mathrm{Co}_{0.45}\right)_{1.5}$ Thin Films
}

\author{
Le Tuan Tu${ }^{1}$, Nguyen Huu Duc ${ }^{2}$, Jong-Ryul Jeong ${ }^{1}$, and CheolGi Kim ${ }^{1 *}$ \\ ${ }^{1}$ Department of Materials Science and Engineering, Chungnam National University, Deajeon 305-764, Korea \\ ${ }^{2}$ Laboratory for Nano Magnetic Materials and Devices, College of Technology, Vietnam National University, \\ Xuan Thuy, Caugiay, Hanoi, Vietnam
}

(Received 28 October 2008, Received in final form 26 November 2008, Accepted 1 December 2008)

\begin{abstract}
In this work the magnetic anisotropies of magnetostriction material $\mathrm{Tb}\left(\mathrm{Fe}_{0.55} \mathrm{Co}_{0.45}\right)_{1.5}$ (named a-TerfecoHan) films were investigated with respect to working pressures in the range 1-7 mTorr. The results obtained show that perpendicular magnetic anisotropy (PMA) can be obtained at a working pressure above 5.1 mTorr. XRD was utilized to clarify the origin of the PMA observed in $\mathrm{Tb}\left(\mathrm{Fe}_{0.55} \mathrm{Co}_{0.45}\right)_{1.5}$ films, and revealed that all samples were amorphous. Therefore, we propose that the PMA effect is explained by stress produced in film due to internal relaxation process and magnetic anisotropy enhancements caused by magnetoelastic interactions.
\end{abstract}

Keywords : magnetostriction, perpendicular magnetic anisotropy, magnetoelastic, TbFeCo

\section{Introduction}

The study of magnetic thin films with perpendicular magnetization is particular important in the in the magnetic recording and sensors industries. Magnetic anisotropy contributions such as, those related to shape, surface, interface interaction, crystalline structure, and strain determine the conditions required for perpendicular magnetization. $\mathrm{R}-\mathrm{Fe}(\mathrm{R}=$ rare earth) based alloys offer the possibility of developing large magnetostrictions at room temperature. When R-Fe compounds are in form of a thin film then amorphous thin films of these alloys generally exhibit extremely high magnetic anisotropies with easy axis perpendicular to the plane of the film [1-3].

The magnetic anisotropy in $\mathrm{TbFeCo}$ originates from interaction between the local electric field and the $4 f$ cloud of $\mathrm{Tb}$ atoms, and its magnitude reflects the asphericity of the $\mathrm{Tb}$ atomic environment. The $4 f$ electronic cloud of $\mathrm{Tb}$ has an oblate ellipsoid shape with a magnetic moment perpendicular to the equatorial plane. When the easy axis of the magnetic moment is perpendicular to the film plane, the minimum electrostatic energy of $4 f$ the cloud occurs when its equatorial plane lies in the plane of the film. Such a configuration should have a minimum

*Corresponding author: Tel: $+82-42-821-6632$

Fax: +82-42-822-6272, e-mail: cgkim@cnu.ac.kr energy as a result of angular dependence of the distance to the different nearest neighbors as well as any nonuniform charge distribution in the equidistant nearestneighbor shell. This aspherical distribution of electric charge, which is the only fundamental physical phenomenon that can be inferred from macroscopic magnetic measurements, has been expressed in terms of different local anisotropies [2].

In the case of $\mathrm{R}-\mathrm{Fe}_{2}$ alloys, the existing phase compounds are $\mathrm{TbFe}_{2}$ (terfenol) and $\mathrm{TbDyFe}_{2}$ (terfenol-D), which exhibit giant positive magnetostriction [4-6]. The $\mathrm{R}-\mathrm{FeCo}$ has also been investigated in the context of magetostriction in amorphous alloys of $\mathrm{Tb}\left(\mathrm{Fe}_{0.55} \mathrm{Co}_{0.45}\right)_{1.5}$ (TerfecoHan), and the TerfecoHan magnetostriction observed was much larger than that observed in other amorphous films of either $\mathrm{TbFe}$ or $\mathrm{TbCo}$ [7-9].

In the present study, we examined factors influenced by working pressure, such as, the magnetic anisotropy of $\mathrm{Tb}\left(\mathrm{Fe}_{0.55} \mathrm{Co}_{0.45}\right)_{1.5}$, and we optimized the working pressure conditions required perpendicular magnetic anisotropy.

\section{Experiment}

Several series of thin films of composition $\mathrm{Tb}\left(\mathrm{Fe}_{0.55^{-}}\right.$ $\left.\mathrm{Co}_{0.45}\right)_{1.5}$ were deposited on $\mathrm{Si}$ wafers by dc-magnetron sputtering at a base pressure of $2 \times 10^{-7}$ Torr and at Ar working pressures in the range 1-7 mTorr. Film thick- 
nesses were $200 \mathrm{~nm}$, which were obtained by controlling sputtering time. During the deposition, a magnetic field of 250 Oe was applied perpendicular film planes to set the unidirectional exchange anisotropy in this direction. All depositions were performed at room temperature.

The magnetic properties of the thin films formed were measured using a Lake shore 7400 series Vibrating Sample Magnetometer (VSM) with the magnetic field applied inplane and perpendicular to the film plane. Anisotropy fields and coercivity were determined from hysteresis loops. Microstructures were determined by X-ray diffractometry using $\mathrm{Cu}-\mathrm{K} \alpha$ radiation. Elemental compositions were determined by EDX (Energy Dispersive X-ray analysis).

\section{Results and Discussion}

Fig. 1 shows the X-ray diffraction patterns of $\mathrm{Tb}\left(\mathrm{Fe}_{0.55^{-}}\right.$ $\left.\mathrm{Co}_{0.45}\right)_{1.5}$ deposited from 1 to 7 mTorr and demonstrates the formation of an amorphous film [8]. EDX was also used to determine elemental compositions of $\mathrm{Tb}\left(\mathrm{Fe}_{0.55^{-}}\right.$ $\left.\mathrm{Co}_{0.45}\right)_{1.5}$ films.

The magnetization curves of these films are shown in Fig. 2, which shows magnetization curves versus internal field $\mathrm{H}_{\text {int }}=\mathrm{H}_{\mathrm{ext}}-\mathrm{NM}$, where $\mathrm{H}_{\mathrm{ext}}, \mathrm{N}$ and $\mathrm{M}$ are external field, demagnetization factor and magnetization, respectively. The demagnetization factor of a thin films is given by $\mathrm{N}=\mathrm{N}_{/}=0$ in-plane and the experimentally determined demagnetization factor in the perpendicular direction is given by $\mathrm{N}=\mathrm{N}_{\perp}$. The value of $\mathrm{N}_{\perp}$ was chosen to correspond to the steepest part of the magnetization curve. The resulting effective values of $\mathrm{N}_{\perp}$ were $1,0.9,0.7$ and 0.5 for deposited films at working pressures of 1,3,5, and 7 mTorr, respectively. As compared with the $\mathrm{N}_{\perp}=1$ expected for an infinite film, obtained value for as-deposited films were too small, which was attributed to the nucleation of

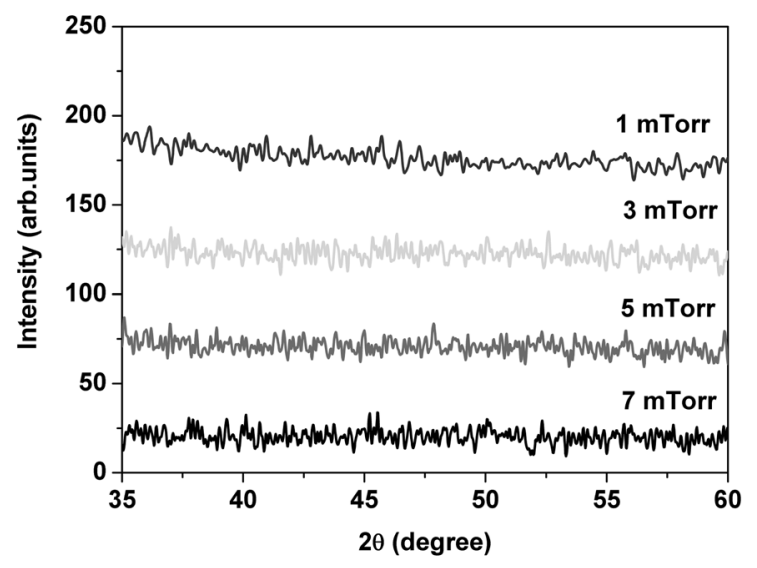

Fig. 1. X-ray diffraction of $\mathrm{Tb}\left(\mathrm{Fe}_{0.55} \mathrm{Co}_{0.45}\right)_{1.5}$ thin films.
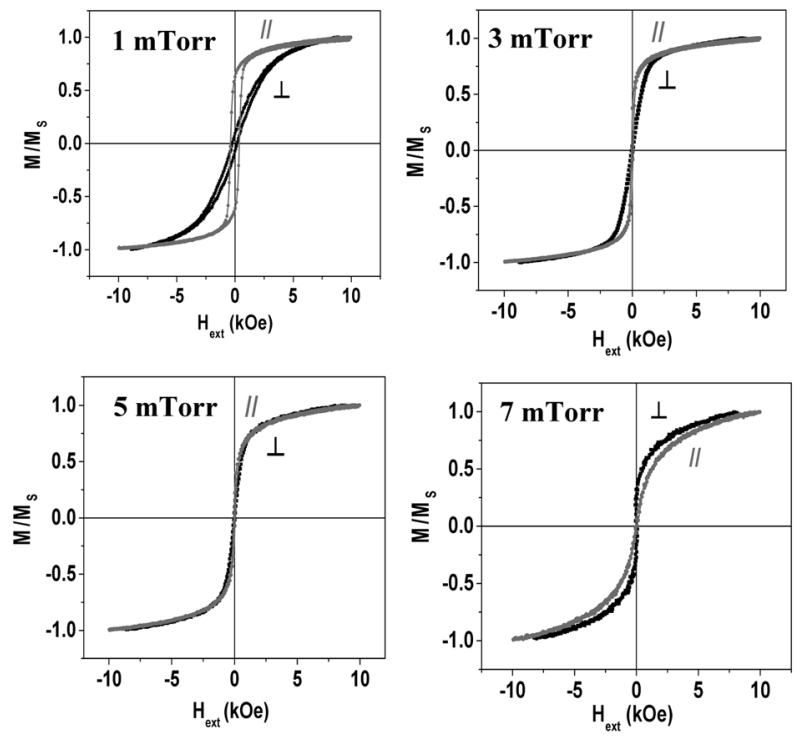

Fig. 2. Hysteresis loops of $\mathrm{Tb}\left(\mathrm{Fe}_{0.55} \mathrm{Co}_{0.45}\right)_{1.5}$ films with magnetic field applied parallel $(/ /)$ and perpendicular $(\perp)$ to the plane of the film.

tripe domains $[8,10,11]$.

$\mathrm{Tb}\left(\mathrm{Fe}_{0.55} \mathrm{Co}_{0.45}\right)_{1.5}$ films have easy magnetization directions changed from in-plane to the perpendicular to the plane. Anisotropy field can be observed from the hysteresis loops (Fig. 2). Effective anisotropy field can be determined using $\mathrm{H}_{\mathrm{k}}=\left(\mathrm{H}_{\mathrm{k} / /}-\mathrm{H}_{\mathrm{k} \perp}\right)$, where $\mathrm{H}_{\mathrm{k} / /}$ and $\mathrm{H}_{\mathrm{k} \perp}$ are the anisotropy fields of in-plane and perpendicular magnetization, respectively $[12,13]$. Effective magnetic anisotropy values were calculated using anisotropy field $\mathrm{K}_{\mathrm{u}}$, defined as $\Delta \mathrm{H}_{\mathrm{k}} . \mathrm{M}_{\mathrm{s}} / 2$, where $\mathrm{M}_{\mathrm{s}}$ is the magnetization [13].

Fig. 3 provides uniaxial anisotropy values as a function of working pressure. When working pressures were above $5.1 \mathrm{mTorr}$ anisotropies were positive values and perpendicular, and at working pressure is below 5.1 mTorr anisotropies in-plane were negative.

At positive $\mathrm{K}_{\mathrm{u}}$ values (perpendicular anisotropy), materials with magnetoelastic anisotropy compensate for external

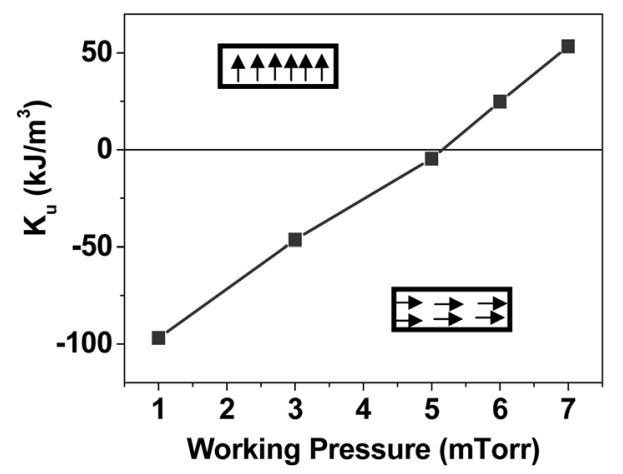

Fig. 3. Dependences uniaxial anisotropy, $\mathrm{K}_{\mathrm{u}}$, on the working pressure of $\mathrm{Tb}\left(\mathrm{Fe}_{0.55} \mathrm{Co}_{0.45}\right)_{1.5}$ films. 


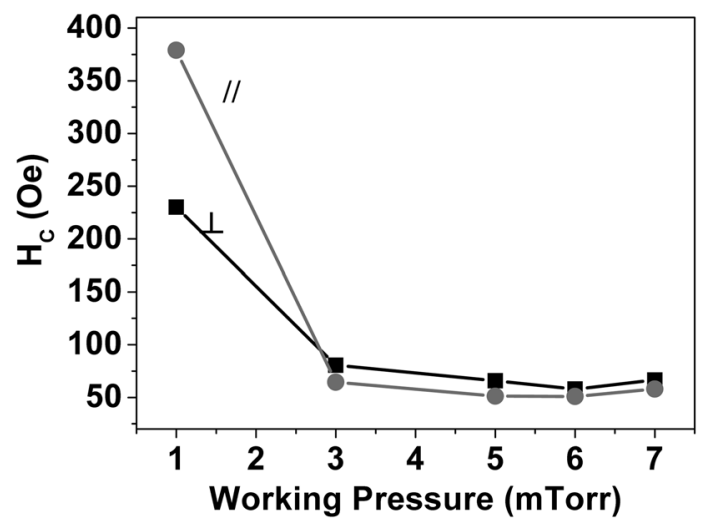

Fig. 4. Coercivity of $\mathrm{Tb}\left(\mathrm{Fe}_{0.55} \mathrm{Co}_{0.45}\right)_{1.5}$ thin films as function of working pressure with magnetic filed applied parallel $(/ /)$ and perpendicular $(\perp)$ to the plane of the film.

or internal stresses by altering spin rotations. For a film with positive magnetostriction, tensile stress leads to spin orientation in the plane of the film, whereas compressive stress causes spins to orientate perpendicularly along the film plane. Since the thermal expansion coefficients of $\mathrm{Tb}-\mathrm{FeCo}$ film and the $\mathrm{Si}$ substrate result in in-plane anisotropy, it is possible that the observed perpendicular anisotropy is of intrinsic origin, and that it is associated with the structural anisotropy induced during the sputtering process [8]. On the other hand, the perpendicular anisotropy of Tb-FeCo film can also be explained theoretically using the "initial-susceptibility method" by determined magnetostriction value [2]. The relationship between anisotropy and magnetostriction is given by: $\chi=$ $\mu_{0} M_{S}^{2} / 2 K-3 \lambda_{S} \sigma$. Here, $\chi, \mathrm{M}_{\mathrm{S}}, \mathrm{K}, \lambda_{\mathrm{S}}$, and $\sigma$ are susceptibility, saturation magnetization, magnetic anisotropy, saturation magnetostriction, and applied tensile stress, respectively $[2,7]$.

The elimination of the perpendicular anisotropy at low working pressure (below $5.1 \mathrm{mTorr}$ ) are isotropic amorphous structure has a lower energy than the as-deposited anisotropic state. Thus, the relaxation of anisotropy without crystallization involves a simple relaxation of the amorphous structure and results in a more stable and homogenous film structure [8].

Coercivity of $\mathrm{Tb}\left(\mathrm{Fe}_{0.55} \mathrm{Co}_{0.45}\right)_{1.5}$ films depends on working pressure (Fig. 4) and its coercivity field is lower than that reported in the literature (Ref. 8). Furthermore, its coercivity is strongly affected by internal stress, microstructure, and homogeneity [14].

\section{Conclusion}

The hysteresis loops obtained using VSM show that perpendicular anisotropy can be observed in $\mathrm{Tb}\left(\mathrm{Fe}_{0.55^{-}}\right.$ $\left.\mathrm{Co}_{0.45}\right)_{1.5}$ amorphous thin films produced by sputtering at a working pressure $>5.1 \mathrm{mTorr}$. The effective anisotropies of amorphous thin films were estimated by calculating uniaxial anisotropies.

\section{Acknowledgment}

The authors greatly acknowledge the financial support from KOSEF under the project number M1080300142708M0300-42710 and from ETRI under project number 2006-S-074-02.

\section{References}

[1] A. Forkl, M. Hirscher, T. Mizoguchi, and H. Kronmiiller, J. Magn. Magn. Mater. 93, 261 (1991).

[2] J. Huang, C. Prados, J. E. Evetts, and A. Hernando. Phys. Rew. B 51, 297 (1995).

[3] N. H. Duc, K. Mackay, J. Betz, and D. Givord, J. Appl. Phys. 87, 834 (2000).

[4] M. I. Batashevich, H. A. Katori, T. Goto, H. Wada, T. Maeda, T. Mori, and M. Shiga, Physica B 229, 315 (1997).

[5] T. Goto, H. A. Katori, T. Sakakibara, H. Mitamura, K. Fukamichi, and K. Murata, J. Appl. Phys. 76, 6682 (1994).

[6] T. Goto, K. Fukamichi, and H. Yamada, Physica B 300, 167 (2001).

[7] N. H. Duc and P. E. Brommer, in: K. H. J. Buschow (Ed.), Handbook on Magnetic Materials, Vol. 14, Elsevier, Amsterdam, p. 89 (2002).

[8] T. M. Danh, N. H. Duc, H. N. Thanh, and J. Teilet, J. Appl. Phys. 87, 7208 (2000).

[9] N. H. Duc, K. Mackay, J. Betz, and D. Givord, J. Appl. Phys. 79, 973 (1996).

[10] K. Ried, M. Schnell, F. Schatz, M. Hirscher, B. Ludescher, W. Sigle, and H. Kronmuller, Phys. Status Solidi A 167, 195 (1998).

[11] A. Forkl, M. Hirscher, T. Mizoguchi, H. Kronmuller, and H. U. Habermeier, J. Magn. Magn. Mater. 93, 261 (1991).

[12] Sebastiaan van Dijken, Jerome Moritz, and J. M. D. Coey, J. Appl. Phys. 97, 063907 (2005).

[13] E. T. de Laceisserie, D. Gignoux, and M. Schlenker, Magnetism, Kluwer Academic Publishers (2002).

[14] F. Hellman, M. Messer, and E. N. Abarra, J. Appl. Phys. 86, 1047 (1999).

[15] L. Jin, S. S. Yoon, P. Kollu, C. G. Kim, D. S. Suhr, and C. O. Kim, J. Magnetics 12(1), 31 (2007).

[16] S. D. Choi, S. W. Kim, D. H. Jin, and D. K. Yun et al., J. Magnetics 11(2), 66 (2006). 